\title{
Modulation Classification Based on Extensible Neural Networks
}

\author{
Guan Qing Yang \\ College of Electronic and Information Engineering, Shenyang Aerospace University, Shenyang 110136, China \\ Correspondence should be addressed to Guan Qing Yang; guan_qy@163.com
}

Received 13 April 2017; Revised 3 August 2017; Accepted 29 August 2017; Published 9 October 2017

Academic Editor: Paolo Crippa

Copyright (C) 2017 Guan Qing Yang. This is an open access article distributed under the Creative Commons Attribution License, which permits unrestricted use, distribution, and reproduction in any medium, provided the original work is properly cited.

\begin{abstract}
A deep learning architecture based on Extensible Neural Networks is proposed for modulation classification in multipath fading channel. Expanded Neural Networks (ENN) are established based on energy natural logarithm model. The model is set up using hidden layers. Modulation classification based on ENN is implemented through the amplitude, phase, and frequency hidden network, respectively. In order to improve Probability of Correct classification (PCC), one or more communication signal features are extracted using hidden networks. Through theoretical proof, ENN learning network is demonstrated to be effective in improving PCC using amplitude, phase, and the frequency weight subnetwork, respectively. Compared with the traditional algorithms, the simulation results show that the proposed ENN has higher PCC than traditional algorithm for modulation classification within the same training sequence and Signal to Noise Ratio (SNR).
\end{abstract}

\section{Introduction}

In the communication transmission technology, modulation classification for communication signals is an important research direction, especially in the field of space communication, satellite communication, underwater communication, and so forth. Modulation classification is constrained by the transmission environmental parameters, such as signal amplitude, signal frequency, and signal phase, especially fading channel.

In the related research literature, there are two kinds of research trends for communication signal modulation classification. One trend is based on the Maximum Likelihood function method based on feature parameter estimation [1]. The other trend is signal statistical features [2]. In order to improve PCC for modulation classification, [3-6] established different Maximum Likelihood (ML) functions. This method was optimized to estimate parameters, such as modulation mode, carrier frequency, phase, and channel response. In [3], a generalized ML function was established. To solve this likelihood function, the QAM signal was verified to obtain lower complexity. In [4], a hybrid ML function was proposed. Instantaneous signal feature was obtained to reduce the complexity. In $[5,6]$, the optimization method based on different
Maximum Likelihood was proposed for MFSK signal modulation. Thus a higher PCC had been obtained. In [7], a hybrid ML algorithm was proposed to classify AMC communication signals.

ML function for modulation classification is generally assisted with Bayesian framework. When the numbers of unknown parameters are too large, the complexity for established likelihood functions would be increasing. Different ML functions and Bayesian decision networks must be evaluated carefully. According to different modulation types, literature [3-7] proposed different likelihood ratio functions, which could obtain the theoretical optimal result under the Gaussian white noise condition. However, multipath fading channel would seriously impact on modulation classification.

Another common method is signal statistical features, which is an easy method to achieve suboptimal result by obtaining statistical characteristics features, such as highorder cumulants, wavelet features, and multidimensional signal features. In [8], an instantaneous signal statistical characteristic was proposed, which included instantaneous amplitude, instantaneous phase, and instantaneous carrier frequency. Reference [9] proposed wavelet feature, which was decomposed into wavelet coefficients by Haar transform. 
Haar transformed wavelet coefficients have better antinoise features than the higher order cumulants.

Literature $[10,11]$ acquired high-order cumulants features. However, higher computation improved the complexity. In [12], HOS statistics feature was obtained, which included time accumulation statistics feature. HOS statistics features had excellent anti-Gaussian white noise. Similarly, the literature [13] adopted HOS features to combat multipath fading. In [14], a statistic classifier within a multipath channel had been proposed to estimate signal parameters. Within multipath fading channel, literature [15] proposed a blind channel estimation method based on higher order statistics. However, this blind channel estimation is only effective in fading channels within two-path channel. In [14], within multipath fading channel, the signal parameters were firstly estimated. In [16], modulation classification in multiuser system was researched. Most of modulation classification algorithms are within Gaussian white noise channel. In fact, due to multipath fading channel, the application for modulation classification algorithms was greatly reduced.

In the past few years, due to rapid development of artificial intelligence, researchers introduced machine learning algorithms into modulation classification and gave the relevant theoretical derivation proof. In [17], the $K$-nearest neighbor clustering classifier was used for AMC modulation classification. Under ideal conditions, better PCC based on $K$-nearest neighbor clustering classifier was only limited to ideal conditions.

In [18], a multilayer neural network was used for the modulation classification. On this basis, literature [19] used ANN neural network for modulation classification. However, the ANN network was only limited to the shallow network. Literature $[18,20]$ implemented modulation classification combined with GA algorithm. On this basis, [17] proposed a KNN algorithm, which was used to improve PCC.

In this paper, a deep learning architecture based on Extensible Neural Networks is proposed for modulation classification in multipath fading channel. Expanded Neural Networks are established through the energy natural logarithm model, which works through the amplitude, phase, and frequency subnetwork, respectively, in order to improve the PCC.

\section{System Model and Problem Formulation}

The Gaussian white noise is according to the Gaussian distribution with mean 0 and variance $N_{0} / 2$, which represents the mean and variance using A/D sampling additive Gaussian white noise (AWGN) signal. Specifically, Gaussian white noise signal is passing through correlated Receive or Matched filter and forming discrete random variable. So we could obtain that the mean is 0 and variance is $N_{0} / 2$. The block diagram end-to-end signal flow chart of the system for ENN is shown in Figure 1.

The received signal passes through ideal matched filter. After A/D sampling, intersymbol interference is cancelled by the equalization. Similarly, the intersymbol interference is also eliminated by ideal symbol equalizer.
For discussion convenience, suppose that the symbol period is less than the channel coherence time. The system adopts ideal channel equalizer to reduce the intersymbol interference.

The received signal $y(t)$ at the $t$ th time gap can be expressed as

$$
y(t)=A \cdot x(t) \exp (j 2 \pi f t+\varphi)+w(t) .
$$

$x(t)$ is defined as the transmitting signal data set for modulation mode $M,\left\{x(1)_{M}, x(2)_{M}, \ldots, x(t)_{M}\right\}, A$ is defined as amplitude gain, $f$ is defined as carrier offset, $\varphi$ is defined as phase deviation, and $w(t)$ is defined as Gauss white noise for power $\sigma_{w}^{2}$.

The received signal $y(t)$ is the input for ENN; suppose that $\widehat{r}(t)=\left[\widehat{r}_{1}(t), \widehat{r}_{2}(t), \ldots, \widehat{r}_{n}(t)\right]$ is the output estimator for the ENN, $\widehat{r}_{l}(t)$ is the output value estimated for the $l$ th order network, and $r_{l}(t)$ is defined as the ideal output value for the $l$ th order network.

Here, a nonlinear logarithmic error energy function has been proposed as the objective function. Suppose that the signal is in the form of polar coordinates, including the amplitude features, phase features, and frequency features of the signal. Define the system's energy loss as $J$ :

$$
J=\sum_{l=1}^{n} \ln \left(\frac{\widehat{r}_{l}(t)}{r_{l}(t)}\right)^{2} .
$$

Similarly, the $l$ th order network output is for estimating the output value, which can be expressed as the exponential energy loss function,

$$
\begin{array}{rl}
\widehat{r}_{l}(t) & =\exp \left(\sum_{k=1}^{K} w_{l k} h_{k}(t)\right) \\
& =\exp \left(\sum_{k=1}^{K}\left|w_{l k}\right| \cdot \exp \left(j 2 \pi f_{w_{l k}} t+\varphi_{w_{l k}}\right) h_{k}(t)\right), \\
l & l=1, \ldots, n .
\end{array}
$$

$w_{l k}$ could be defined as the weight between the $k$ th hidden layer network and the $l$ th output network. The weight amplitude can be expressed as

$$
A_{w}=\left|w_{l k}\right|, \quad l=1, \ldots, n .
$$

The corresponding weight for the phase and frequency can be expressed, respectively, as $f_{w_{l k}}, \varphi_{w_{l k}} . h_{k}$ can be defined as a hidden layer activation function; the hidden layer needs to be obtained by the following activation function, which can be expressed as

$$
\tan \mathrm{h}(y)=e^{y}-\frac{e^{-y}}{e^{y}}+e^{-y} .
$$

Here, the input for ENN is the sequence $y(t)$, which is the received multipath fading signal defined as $y(t)=$ $\left[y_{1}(t), \ldots, y_{m}(t)\right] \in C^{m}$. Thus, the $k$ th hidden layer unit $h_{k}(t)$ can be expressed as

$$
h_{k}(t)=\tanh \left(\lambda_{k}^{T}\left(y(t)-u_{k}\right)\right), \quad k=1, \ldots, K .
$$




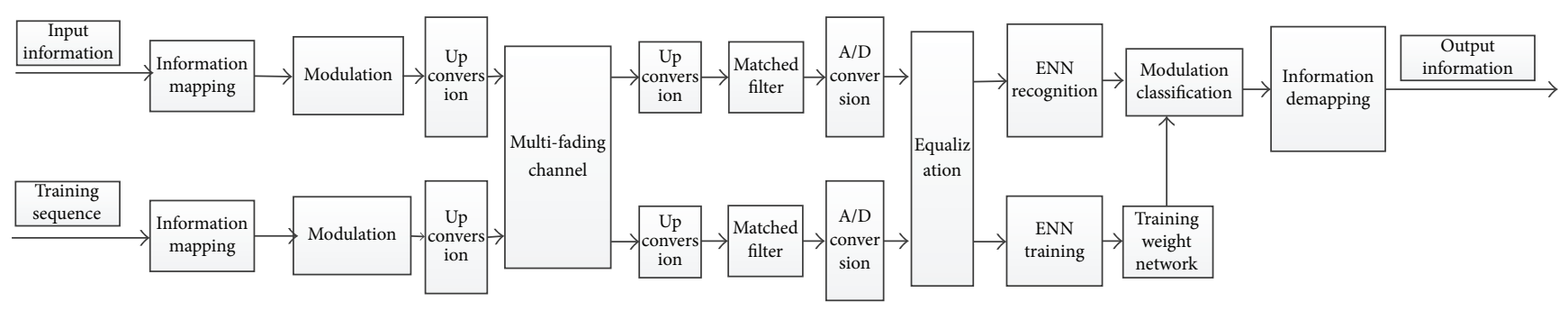

FIGURE 1: The block diagram end-to-end signal processing of the system for ENN.

$\lambda_{k}$ is defined as the error attenuation factor for the $k$ th neural network, which could be expressed as $\lambda_{k}=\left[\lambda_{k}(1), \ldots\right.$, $\left.\lambda_{k}(t)\right]^{T} . u_{k}$ is the $k$ th hidden layer average center processing unit, which can be expressed as $u_{k}=\left[u_{k}(1), \ldots, u_{k}(t)\right] \in C^{m}$.

In this paper, the hidden layer needs to be obtained by the following activation function, which can be expressed as $\tan \mathrm{h}(y)=e^{y}-e^{-y} / e^{y}+e^{-y}$. The activation function needs to be differential everywhere. The condition is as below:

$$
\begin{aligned}
& \forall h^{\prime}, R \rightarrow R \text {, while satisfying } \lim _{n \rightarrow+\infty} h^{\prime}(x)=0 \text { and } \\
& \lim _{n \rightarrow-\infty} h^{\prime}(x)=0 .
\end{aligned}
$$

Currently, the available function is considered as activation function, including the sigmod function and the tanh function. As learning network construction, each layer requires faster convergence. So the ENN select tanh function as an activation function.

Define $\{(r(1), y(2)), \ldots,(r(n), y(n)), \ldots,(r(t), y(t))\}, n \ll$ $t$, as the system network training library, in which $y(n)$ is defined as the input of the $n$th training unit. $r(n)$ is defined as the target output of the $n$th training unit. Define signal error as $\zeta_{r}$, after $l$ th layer network training, which can be defined as

$$
\zeta_{r}=\left|r_{l}(t)-\widehat{r}_{l}(t)\right| \longrightarrow 0 .
$$

Define amplitude error as $\zeta_{A}$, which can be defined as

$$
\zeta_{A}=\left|A_{r}-A_{\widehat{r}}\right| \longrightarrow 0 .
$$

Define phase error as $\zeta_{\varphi}$, which can be defined as

$$
\zeta_{\varphi}=\left|\varphi_{l}-\widehat{\varphi}_{l}\right| \longrightarrow 0 .
$$

Define phase error as $\zeta_{f}$, which can be defined as

$$
\zeta_{f}=\left|r_{f}-r_{\hat{f}}\right| \longrightarrow 0 .
$$

The framework for deep learning ENN is shown as in Figure 2.

The output feature for the $t$ th training sample is defined as $r(t)$. It can be expressed in polar coordinates to obtain amplitude, phase, and frequency information based on energy function model. The signal $r(t)$ can be expressed as

$$
r(t)=A_{r} \exp \left(j 2 \pi f_{r}(t)+\varphi_{r}\right) .
$$

$A_{r}$ is defined as signal amplitude, which also can be expressed as $A_{r}=|r(t)|, \varphi_{r}$ is defined as signal phase, and $f_{r}(t)$ is defined as frequency. Substituting (11) into (2), we could obtain that

$$
J=\sum_{l=1}^{n}\left(\left(\frac{\widehat{r}_{l}(t)}{r_{l}(t)}\right)^{2}+\left(\widehat{\varphi}_{r}-\varphi_{r}\right)^{2}+\left(\widehat{f}_{r}-f_{r}\right)^{2}\right) .
$$

For $N$ training sequences, the target energy function can be defined as

$$
J(W)=\sum_{l=1}^{n}\left(\left(\frac{A_{w l}}{A_{l}}\right)^{2}+\left(\varphi_{w l}-\varphi_{l}\right)^{2}+\left(f_{w l}-f_{l}\right)^{2}\right),
$$

where $W$ is the weight of the network training system. In order to obtain the optimal training objectives, the objective function is minimized, which can be expressed as

$$
J(W) \longrightarrow 0 .
$$

Similarly, the connection weight can be expressed as

$$
W^{*}=\underset{W \in C^{n \times k}}{\arg \min } J(W) .
$$

It could be obtained as

$$
\frac{\partial J(W)}{\partial w_{l}}=0 .
$$

Expanding formula (16), we could obtain

$$
\begin{gathered}
J(W)=\sum_{t=1}^{N} \sum_{l=1}^{n}\left(\ln \left(\widehat{r}_{l}(t)\right)-\ln \left(r_{l}(t)\right)\right)^{2} \\
=\sum_{t=1}^{N} \sum_{l=1}^{n}\left(\ln \left(\widehat{r}_{l}(t)\right)-\ln \left(r_{l}(t)\right)\right) \\
\cdot \overline{\left(\ln \left(\widehat{r}_{l}(t)\right)-\ln \left(r_{l}(t)\right)\right)}
\end{gathered}
$$

Substituting (2) into (17), which is available, we could obtain

$$
\begin{aligned}
& J(W)=\sum_{t=1}^{N} \sum_{l=1}^{n}\left(\sum_{k=1}^{K} w_{l k} h_{k}(t)-\ln \left(r_{l}(t)\right)\right) \\
& \overline{\left(\sum_{k=1}^{K} w_{l k} h_{k}(t)-\ln \left(r_{l}(t)\right)\right)} .
\end{aligned}
$$




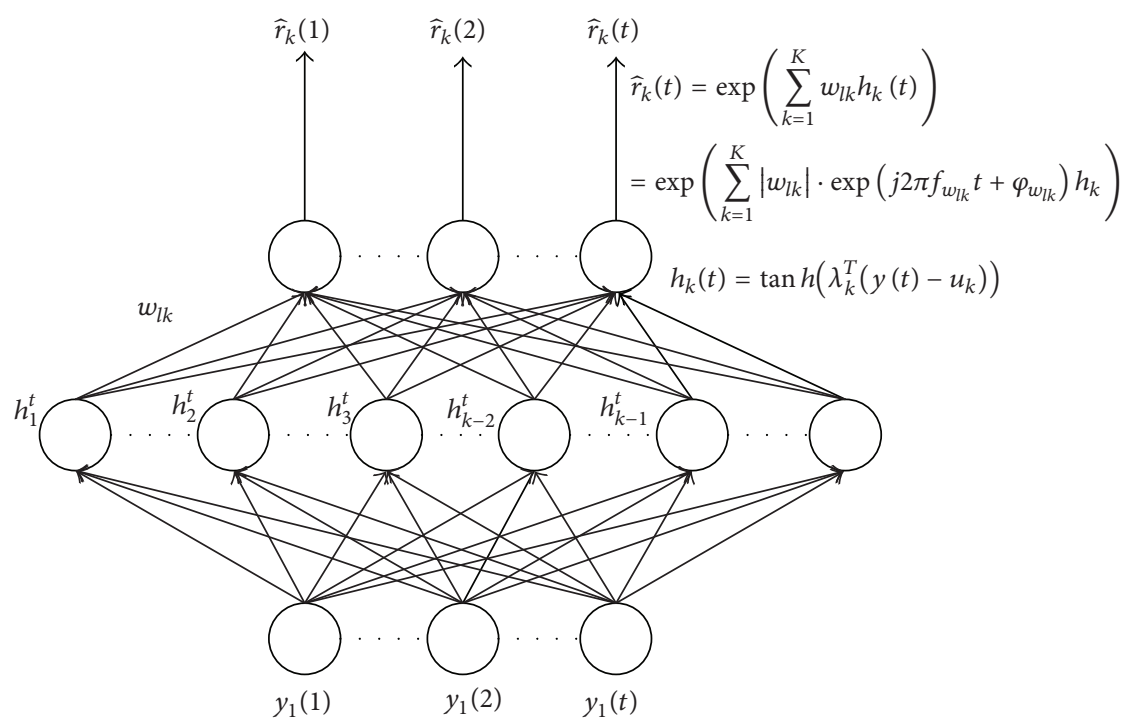

FIGURE 2: Framework for deep learning ENN.

We could obtain the gradient of (18) for $w_{l k}$, and we could obtain

$$
\frac{\partial J(W)}{\partial w_{l}}=\sum_{t=1}^{N} h(t)\left[\sum_{k=1}^{K} \bar{w}_{l k} \bar{h}_{k}(t)-\ln \left(\bar{y}_{l}(t)\right)\right] .
$$

And then we simplified (19) as

$$
\sum_{k=1}^{K} \bar{w}_{l k} \sum_{t=1}^{N} h(t) \bar{h}_{k}(t)=\sum_{t=1}^{N} \ln \left(\bar{y}_{l}(t)\right) h(t) .
$$

The received multipath fading signal enters the different training network through amplitude, phase, and frequency subnetwork, respectively. Among them, the amplitude weight network can be expressed as

$$
\sum_{k=1}^{K} A_{|w|} \sum_{t=1}^{N} h(t) \bar{h}_{k}(t)=\sum_{t=1}^{N} \ln \left(\bar{r}_{l}(t)\right) h(t) .
$$

Among them, the phase weight network can be expressed as

$$
\sum_{k=1}^{K}\left|\varphi_{w l}\right| \sum_{t=1}^{N} h(t) \bar{h}_{k}(t)=\sum_{t=1}^{N} \ln \left(\bar{\varphi}_{l}(t)\right) h(t) .
$$

Among them, the frequency weight network can be expressed as

$$
\sum_{k=1}^{K}\left|f_{w l}\right| \sum_{t=1}^{N} h(t) \bar{h}_{k}(t)=\sum_{t=1}^{N} \ln \left(\bar{f}_{l}(t)\right) h(t) .
$$

The above formulas could be represented by matrix, which can be simplified as

$$
\bar{W} \Pi=\Lambda,
$$

where

$$
\begin{aligned}
& \Pi_{k}=\sum_{t=1}^{N} h(t) \bar{h}_{k}, \quad k=1, \ldots, n, \\
& \Lambda_{l}=\sum_{t=1}^{N} \ln \bar{r}_{l}(t) h(t), \quad l=1, \ldots, n .
\end{aligned}
$$

In accordance with different training network, the matrix form for amplitude can be expressed as

$$
\left|\bar{W}_{A}\right| \Pi=\Lambda \text {. }
$$

The matrix form for phase can be expressed as

$$
\bar{W}_{\varphi} \Pi=\Lambda \text {. }
$$

The matrix form for frequency can be expressed as

$$
\bar{W}_{f} \Pi=\Lambda \text {. }
$$

According to formulas (24)-(28), we could obtain the learning network as shown in Figure 3.

\section{ENN Algorithm}

According to Figure 2, the network of ENN algorithm can be implemented as follows.

Algorithm 1 (network learning based on amplitude information). Given a training set, $\{(y(1), r(1)),(y(2), r(2)), \ldots$, $(y(t), r(t)), \ldots\}$, one has the following.

Select the $K$ layer hidden layer network for training, in which the hidden layer parameters are $u_{k}, v_{k} ; k=1, \ldots, K$.

Calculating the $k$ th hidden layer response, $h_{k}(t)$,

$$
h_{k}(t)=\tanh \left(\lambda_{k}^{T}\left(y(t)-u_{k}\right)\right), \quad k=1, \ldots, K \text {. }
$$




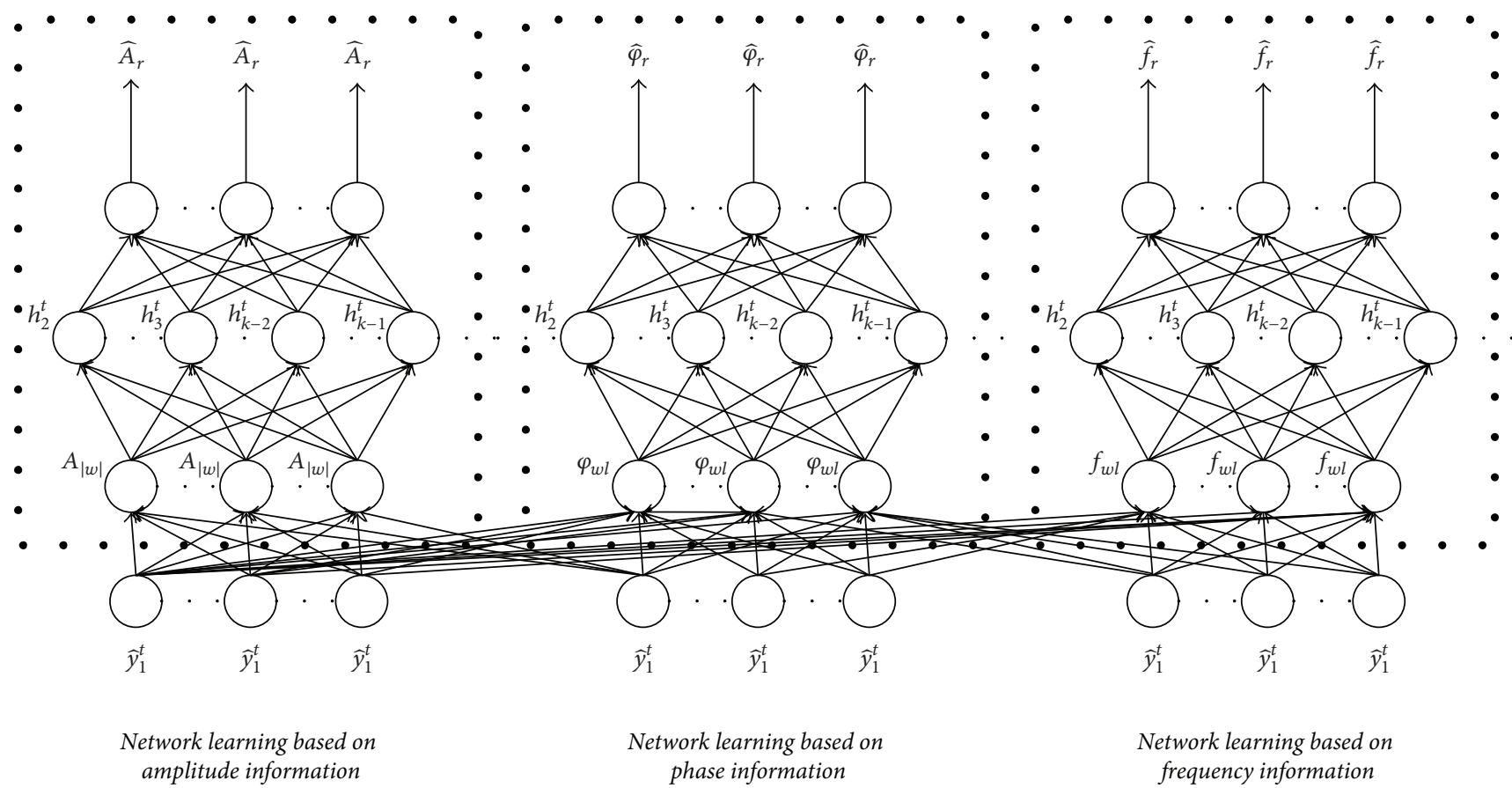

FIGURE 3: Diagram for ENN depth learning network.

Calculate the front matrix $\Pi$,

$$
\Pi_{k}=\sum_{t=1}^{N} h(t) \bar{h}_{k}, \quad k=1, \ldots, n .
$$

Calculate the posterior matrix $\Lambda$,

$$
\Lambda_{l}=\sum_{t=1}^{N} \ln \bar{r}_{l}(t) h(t), \quad l=1, \ldots, n .
$$

Calculate the network weight matrix,

$$
\left|\bar{W}_{A}\right| \Pi=\Lambda \text {. }
$$

Algorithm 2 (network learning based on phase information). Given a training set, $\{(y(1), r(1)),(y(2), r(2)), \ldots$, $(y(t), r(t)), \ldots\}$, one has the following.

Select the $K$ layer hidden layer network for training, in which the hidden layer parameters are $u_{k}, v_{k} ; k=1, \ldots, K$.

Calculating the $k$ th hidden layer response, $h_{k}(t)$,

$$
h_{k}(t)=\tanh \left(\lambda_{k}^{T}\left(y(t)-u_{k}\right)\right), \quad k=1, \ldots, K .
$$

Calculate the front matrix $\Pi$,

$$
\Pi_{k}=\sum_{t=1}^{N} h(t) \bar{h}_{k}, \quad k=1, \ldots, n .
$$

Calculate the posterior matrix $\Lambda$,

$$
\Lambda_{l}=\sum_{t=1}^{N} \ln \bar{r}_{l}(t) h(t), \quad l=1, \ldots, n .
$$

Calculate the network weight matrix,

$$
\bar{W}_{\varphi} \Pi=\Lambda \text {. }
$$

Algorithm 3 (network learning based on frequency information). Given a training set, $\{(y(1), r(1)),(y(2), r(2)), \ldots$, $(y(t), r(t)), \ldots\}$, one has the following.

Select the $K$ layer hidden layer network for training, in which the hidden layer parameters are $u_{k}, v_{k} ; k=1, \ldots, K$.

Calculating the $k$ th hidden layer response, $h_{k}(t)$,

$$
h_{k}(t)=\tanh \left(\lambda_{k}^{T}\left(y(t)-u_{k}\right)\right), \quad k=1, \ldots, K .
$$

Calculate the front matrix $\Pi$,

$$
\Pi_{k}=\sum_{t=1}^{N} h(t) \bar{h}_{k}, \quad k=1, \ldots, n .
$$

Calculate the posterior matrix $\Lambda$,

$$
\Lambda_{l}=\sum_{t=1}^{N} \ln \bar{r}_{l}(t) h(t), \quad l=1, \ldots, n .
$$

Calculate the network weight matrix,

$$
\bar{W}_{f} \Pi=\Lambda \text {. }
$$

\section{Experimental Classification Results and Analysis}

The network of deep learning ENN is established for modulation classification. The modulation consists of BPSK, QPSK, 8PSK, and QAM. The signal modulation mode is generated by a random generator. Signal carrier frequency is defined as $f_{c}=100 \mathrm{KHz}$. Sampling rate is defined as $f_{s}=4 f_{c}=$ $400 \mathrm{KHz}$ 


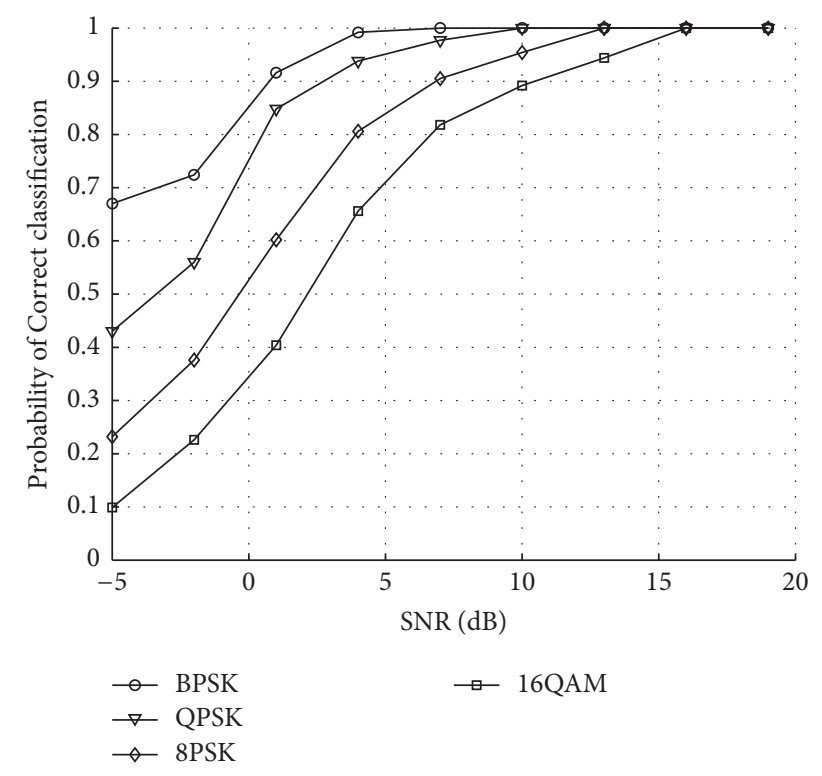

FIGURE 4: Probability of Correct classification for ENN based on different modulation.

The experiment is simulated by Monte Carlo method. SNR ranges from $-5 \mathrm{~dB}$ to $20 \mathrm{~dB}$. Suppose that 512 signal samples are constructed into one data frame, and there are 1000 sets. The first 100 sets are used as training, and 900 sets left are used for testing.

Channel model has been used corresponding to a typical multipath model shown in Table 1, which is modeled as Rayleigh fading channels. According to the three-diameter model, the main channel is adopted as the Rice channel model, and the second-diameter model is adopted as the Rayleigh channel model.

In order to simplify the simulation, we set ENN learning network using three subnetworks, each subnetwork including 30 layers. The number of hidden nodes is defined as 100 . In this paper, we use the HML algorithm [7], comparison with the ANN algorithm based on the constant modulus statistics algorithm [14], and literature [19]. We demonstrate the performance based on the PCC under different SNR, different training length, and the convergence performance.

4.1. Classification for Different Modulation Modes with ENN Deep Learning Network. Define that the SNR varies from $5 \mathrm{~dB}$ to $15 \mathrm{~dB}$, and each of the 512 simulation signal samples forms a data frame. Figure 4 shows the PCC based on BPSK, QPSK, 8PSK, and 16QAM at different SNR. When the SNR is greater than $0 \mathrm{~dB}$, the PCC is above $93 \%$. When the SNR is greater than $10 \mathrm{~dB}, \mathrm{PCC}$ for all modulation methods is more than $80 \%$.

Figure 5 shows the PCC based on BPSK, QPSK, 8PSK, and 16QAM modulation signals at different SNR. Define different modulation modes occurring at equal probability. Suppose that SNR ranges from $-5 \mathrm{~dB}$ to $20 \mathrm{~dB}$, and 1024 signal sampling points constitute a data frame. The number of data frames is set as 1000. Different algorithms are carried out according to different training lengths. Define the length of

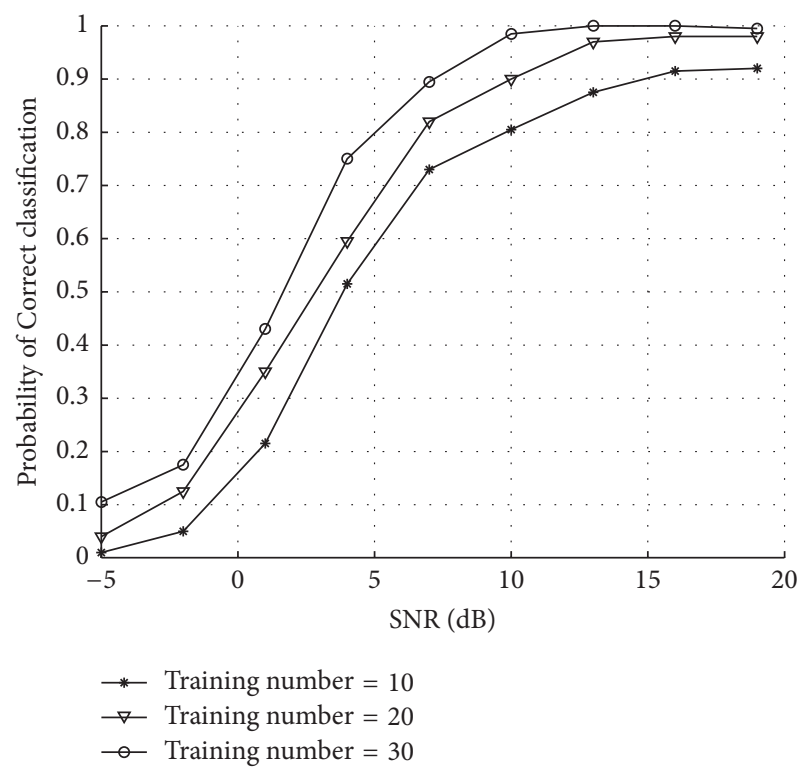

Figure 5: Probability of Correct classification for ENN based on different lengths of training sequences.

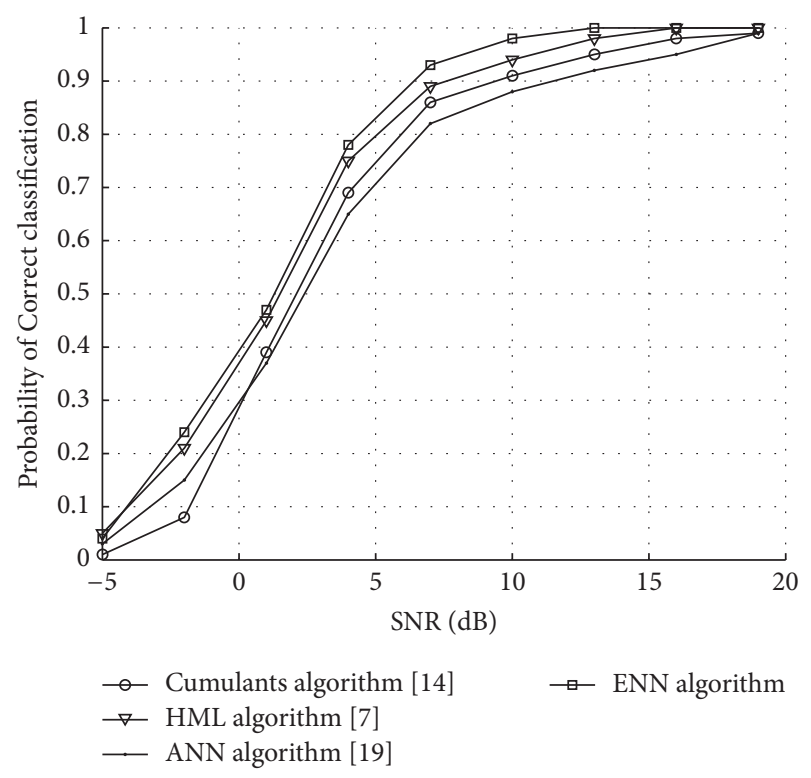

FIGURE 6: Probability of Correct classification simulation based on different Signal to Noise Ratio for different algorithm.

the training symbols, respectively, $N=10,20$, 30. Enough training lengths can effectively improve the PCC. Under the same PCC, the distance for different training lengths can be nearly $3 \mathrm{~dB}$.

4.2. PCC Simulation Based on Different Signal to Noise Ratio. Figure 6 shows the PCC performance of ENN, and algorithms in literature $[7,14,19]$ for comparison at SNR ranged from the $-5 \mathrm{~dB}$ to $20 \mathrm{~dB}$. Suppose that all the algorithms have adopted the same training sequence, $N=20$; when the $\mathrm{SNR}=5 \mathrm{~dB}$, the PCC is below $90 \%$. The performance of the 
TABLE 1: Channel model parameter.

\begin{tabular}{|c|c|c|c|c|c|}
\hline Tap & Distribution function & Parameter & Parameter distribution & Numerical value/dB & Time delay/ns \\
\hline (1) & LOS : Rician & Rice factor & $K$ & 6.3 & 0 \\
\hline (2) & Rayleigh & Average multipath power & $2 \sigma_{l}^{2}$ & -24.1 & 100 \\
\hline (3) & Rayleigh & Average multipath power & $2 \sigma_{l}^{2}$ & -25.2 & 250 \\
\hline
\end{tabular}

algorithm is greatly affected by noise. When SNR $=10 \mathrm{~dB}$, the PCC is increasing. In the multipath fading channel, with the increasing of SNR, the overall performance of different algorithms is closer to the ideal value. The proposed ENN algorithm uses hierarchical identification, and the overall PCC is higher.

HML algorithm has established the likelihood function model in the Gaussian white noise, but the PCC has greatly impacted on the conditions of fading channel. The cumulants of amplitude are affected by the fading multipath channel, especially under low SNR condition. The proposed deep learning ENN algorithm improves the fitness for the amplitude in the fading channel, which is implemented by the learning of the amplitude weight subnetwork, especially within the slow fading channel.

In addition, PCC of HML increases rapidly with SNR increasing. HML has better classification ability. However, HML algorithm performance is constrained by multipath fading channels. Thus the performance has not shown advantages. It can be seen from the simulation results that the proposed algorithm has high accuracy and stability under different SNR. Compared with the traditional neural network ANN algorithm, the proposed algorithm has higher PCC and reliability. This is because the deep learning ENN algorithm is based on signal features, which is based on different dimensions of subnetwork identification. The overall performance has shown more advantages. In the SNR at more than $5 \mathrm{~dB}$, the PCC can reach more than $90 \%$.

\subsection{Probability of Correct Classification Simulation Based on} Different Lengths of Training Sequences. In order to demonstrate the performance of different modulation classification algorithms with different training samples, suppose that the 1024 signal sampling points constitute a data frame, and the total number for classified data frames is identified as 1000 according to the different training length.

Figure 7 shows the PCC performance under the condition of training samples from $N=5$ to $N=40$ at $\mathrm{SNR}=$ $5 \mathrm{~dB}$. We can obtain from the simulation figure that, as the training number increases, the PCC is near optimal. When the number of samples is $N=25$, the PCC is higher than $90 \%$. When the training number is small, the neural network ANN structure weight for training $W$ is not sufficient, so the performance is poor. The cumulants algorithm has low performance, mainly because of the feature extraction based on amplitude feature, which reduces the performance in multipath channel.

The ML is based on the likelihood criterion and makes full use of the estimation about unknown parameters. Therefore, under the same training number condition, the performance

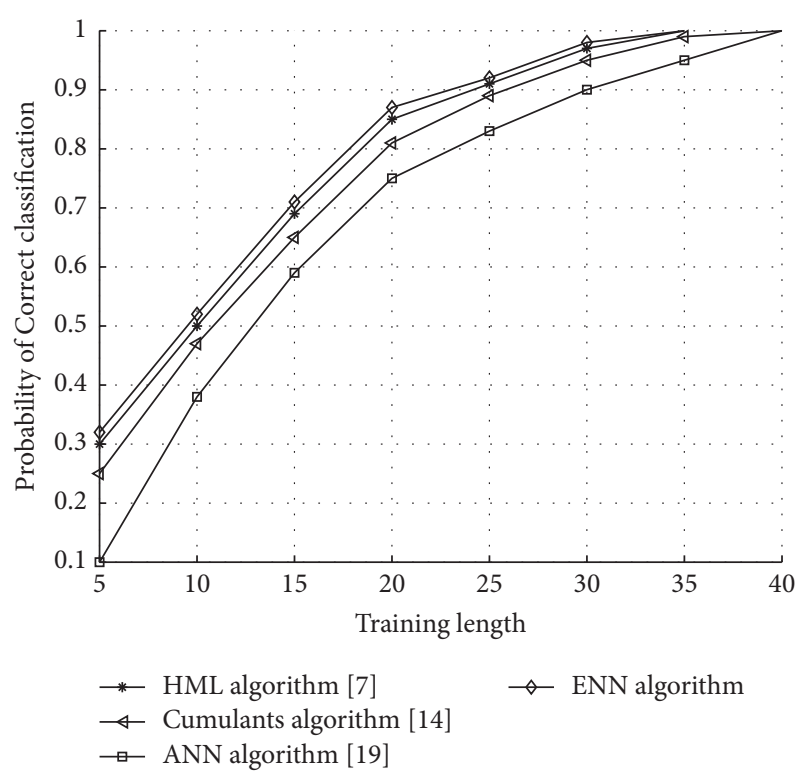

FIGURE 7: Probability of Correct classification simulation based on different lengths of training sequences for different algorithm.

is higher than ANN algorithm. The proposed ENN algorithm has higher accuracy and stability, with high reliability and better performance advantages. In the case of small sample $N=25$ group, the correct classification rate can reach more than $90 \%$. Thus, the proposed ENN also has excellent classification performance with small sample. The PCC of the signal modulation mode is higher than the literature algorithm $[7,14,19]$.

4.4. Convergence Performance Simulation Based on Different Algorithm. Figure 8 shows the convergence performance of the proposed deep learning network ENN and ANN classifier algorithm proposed in [19] within different training sequences. With the length of the training sequence increasing, the ENN algorithm has faster convergence performance. This is mainly because the ENN algorithm has faster convergence using multidimensional features and quickly enters fast convergence direction. Therefore, the convergence performance of the ENN algorithm can be improved rapidly. In addition, when the number of training sequences is small, ENN classifier algorithm performance is better than ANN algorithm according to PCC. So the proposed ENN algorithm has better adaptability with small training sequences. Compared with the existing literature [19], the ENN algorithm proposed in this paper has better classification performance and has a better training sequence dynamic range. 


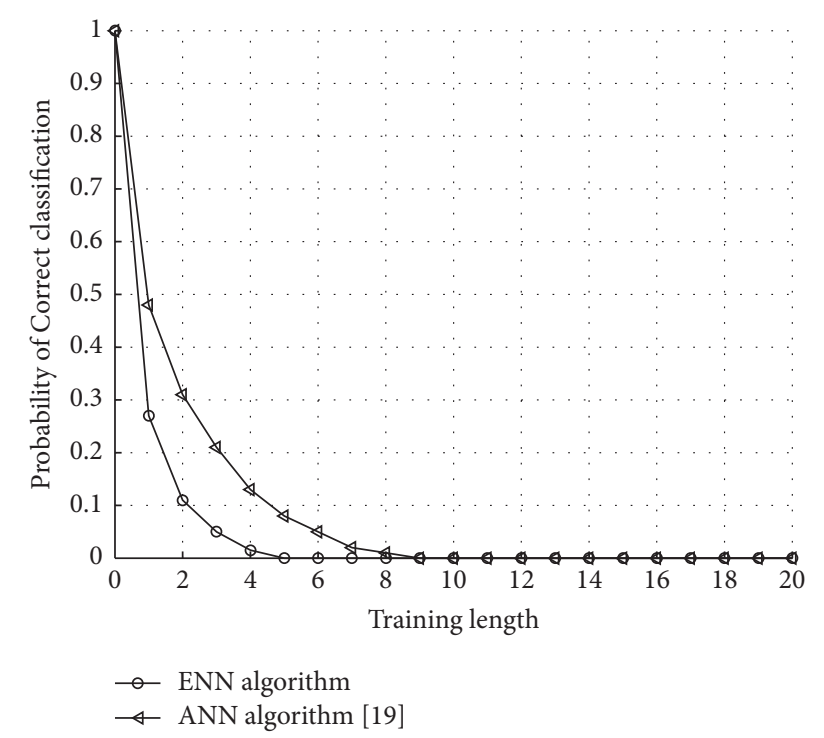

FIGURE 8: Convergence performance simulation based on different algorithm.

4.5. Probability of Correct Classification Simulation Based on MIMO-OFDM. In order to analyze the modulation classification for OFDM, the communication system based on OFDM modulation is established. In the simulation, establishing a 2-channel channel, the main path has a direct component. The second path has a fading rate of 0.4 with a maximum delay $180 \mathrm{~ns}$. Amplitude fading is supposed as Rician distribution. The link transmission bit rate is $40 \mathrm{Mbit} / \mathrm{s}$. The frame length is 16384 bits.

Suppose that the cyclic prefix length of OFDM is larger than the maximum spreading delay; the guard interval should be 250 ns multiplied by 4 , which is 1 us. Then the symbol period of OFDM can be 6 us. So each OFDM symbol needs to be transmitted with $(40 \mathrm{Mbit} / \mathrm{s}) /(1 / 6 \mathrm{us})=240 \mathrm{bits}$. OFDM symbols within each subcarrier can be transmitted with 2 bits, which should need $240 / 2=120$ subcarriers to meet with transmission rate requirements. The zero-padded subcarriers should be added to eight digit number to facilitate the realization of 128-point FFT/IFFT. For the OFDM modulation, the normalized frequency offset factor is used to measure frequency offset. The normalized frequency offset factor is defined as the ratio of the carrier frequency deviation to the subcarrier spacing. Normally, if the normalized frequency offset is an integral, the orthogonality for subcarrier will not be destroyed.

The orthogonality for subcarrier will be destroyed. When the frequency deviation of the subcarrier is 0.5 times for the carrier interval, the orthogonality of the subcarrier will be minimized, resulting in serious loss of demodulation signal performance. The OFDM system can obtain a carrier frequency interval of approximately 0.5 times the frequency deviation, it will seriously damage the subcarrier orthogonality, and the demodulation of the signal performance has a great impact.

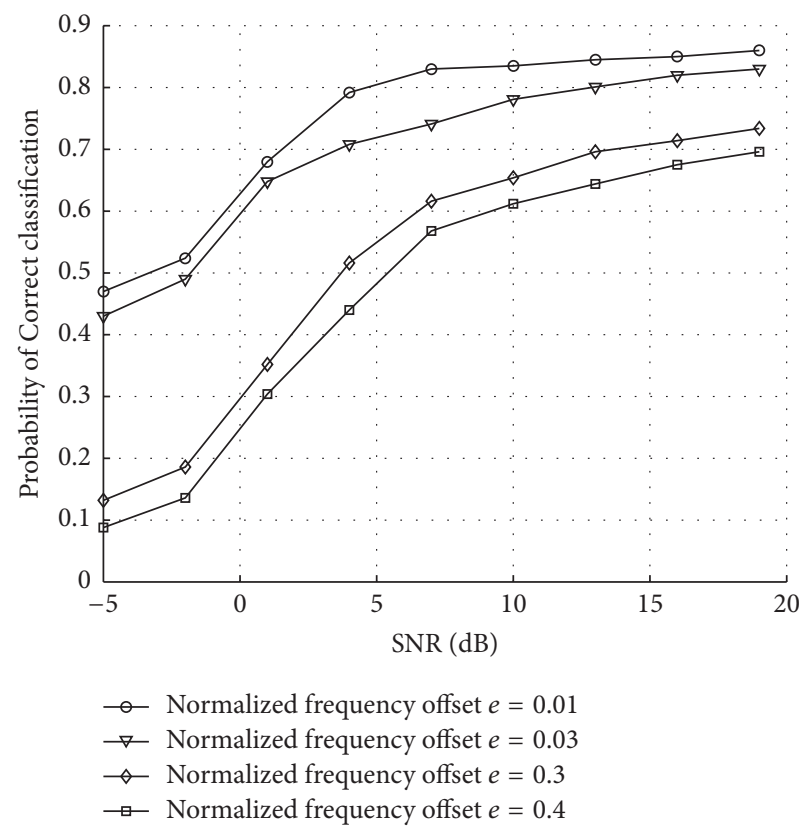

Figure 9: Probability of Correct classification for ENN based on different normalized frequency offsets.

The paper is mainly for the normalized frequency offset factor within 0.5 times. For the deviation analysis, the normalized frequency offset factor is set as $e=0.01, e=0.03$, $e=0.3, e=0.35$.

It can be obtained from the simulation in Figure 9, when the normalized frequency offset factor $e \leq 0.3$, which is lower, the PCC for ENN is lower due to the different normalized frequency offsets. This is because the proposed ENN has correction capability for the small frequency deviation. When the normalized frequency offset $e>0.3$, the frequency offset has introduced a larger orthogonal subcarrier failure. For the signal modulation classification, the error has exceeded the relevant decision limits, and PCC is low.

It can be obtained from the simulation in Figure 9, when the normalized frequency offset factor $e \leq 0.3$, which is lower, the PCC for ENN is lower due to the different normalized frequency offsets. This is because the proposed ENN has some error correction capability for the small frequency deviation. When the normalized frequency offset $e>0.3$, the frequency offset has introduced a larger orthogonal subcarrier failure. For the signal modulation classification, the error has exceeded the relevant decision limits, and the PCC is low.

4.6. Probability of Correct Classification Simulation Based on MIMO. Based on OFDM modulation mode link communication, the antenna model is extended from SISO to MIMO. MIMO adopts $2 \times 2$ and $4 \times 4$ antenna group, respectively, to establish STBC coding mode. The PCC for OFDM-MIMO has been analyzed. In the simulation, a 2-channel channel is used. The main path has a direct component, and the amplitude fading follows the Rician distribution. The fading amplitude for the second diameter is 0.4 for the first diameter, 


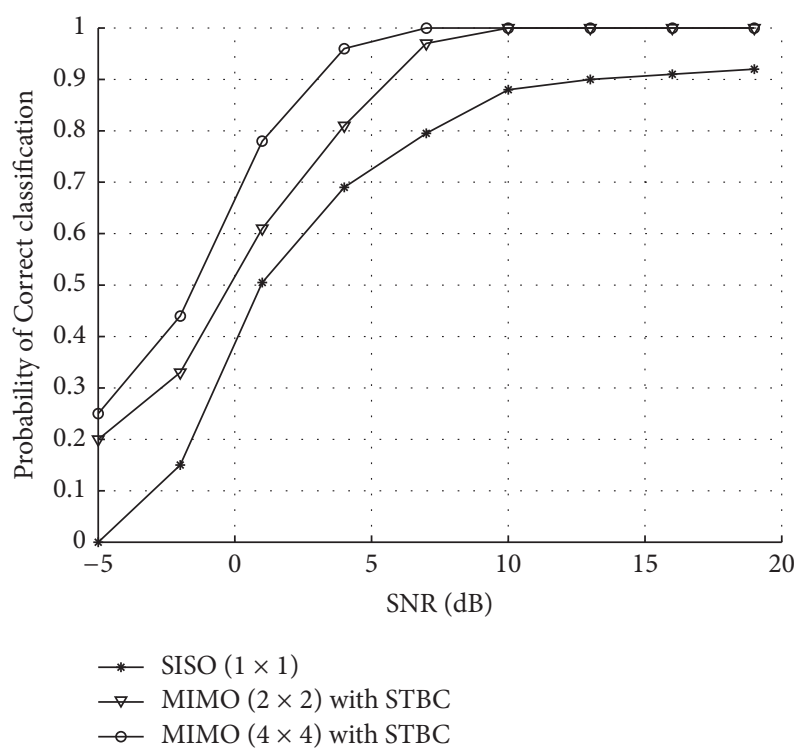

FIgURE 10: Probability of Correct classification for ENN based on different antennas array.

the maximum delay is $180 \mathrm{~ns}$, the channel bandwidth is $10 \mathrm{MHz}$, and the frame length is 128-symbol cycle.

From the simulation in Figure 10, we can obtain that MIMO-STBC system can combat with a certain channel fading characteristic. The ENN with MIMO-STBC can be used to improve the Probability of Correct classification. Therefore, combined with the STBC coding of MIMO system, the proposed ENN depth modulation pattern recognition network can obtain high PCC.

4.7. Complexity Analysis of Different Algorithms. The proposed ENN deep learning network performs the best performance because of sacrificing the complexity, and the multiple identifiers are trained by different signal features including amplitude, phase, and frequency and are judged by the combined features. So the PCC results are available in both small sample and low SNR conditions.

Viewing the classification time with the same experimental environment, the constant modulus statistics algorithm has the faster velocity because of only extracting the amplitude features information. Followed by the ANN network, ANN network needs to establish the neural networks through the amplitude characteristics.

\section{Conclusion}

In this paper, an adaptive Extensible Neural Network has been proposed for modulation classification in the multipath fading channel. By establishing the natural logarithmic energy model of the desired model, the deep learning ENN has been divided into the amplitude, phase, and frequency weight subnetwork. Compared with the traditional algorithm, the simulation results show that the proposed ENN algorithm has efficient classification performance and small sample classification ability and could be adaptive in multipath fading channels with the same length training sequence and SNR.

\section{Conflicts of Interest}

The author declares that they have no conflicts of interest.

\section{Acknowledgments}

The work reported here is supported by National Natural Science Foundation of China (no. 61501306), The Natural Science Foundation of Liaoning Province of China (no. 2015020026), and The Natural Science Foundation of Liaoning Provincial Department of Education (no. L2015402).

\section{References}

[1] J. L. Xu, W. Su, and M. Zhou, "Likelihood-ratio approaches to automatic modulation classification," IEEE Transactions on Systems, Man and Cybernetics Part C: Applications and Reviews, vol. 41, no. 4, pp. 455-469, 2011.

[2] W. Su, "Feature space analysis of modulation classification using very high-order statistics," IEEE Communications Letters, vol. 17, no. 9, pp. 1688-1691, 2013.

[3] O. A. Dobre, A. Abdi, Y. Bar-Ness, and W. Su, "Blind modulation classification: a concept whose time has come," in Proceedings of the IEEE/Sarnoff Symposium on Advances in Wired and Wireless Communication, pp. 223-228, April 2005.

[4] F. Hameed, O. A. Dobre, and D. C. Popescu, "On the likelihoodbased approach to modulation classification," IEEE Transactions on Wireless Communications, vol. 8, no. 12, pp. 5884-5892, 2009.

[5] B. F. Beidas and C. L. Weber, "Asynchronous classification of mfsk signals using the higher order correlation domain," IEEE Transactions on Communications, vol. 46, no. 4, pp. 480-493, 1998.

[6] A. E. El-Mahdy and N. M. Namazi, "Classification of multiple $\mathrm{M}$-ary frequency-shift keying signals over a Rayleigh fading channel," IEEE Transactions on Communications, vol. 50, no. 6, pp. 967-974, 2002.

[7] O. Ozdemir, P. K. Varshney, and W. Su, "Asynchronous hybrid maximum likelihood classification of linear modulations," in Proceedings of the 2013 IEEE Global Communications Conference, GLOBECOM 2013, pp. 3235-3240, December 2013.

[8] A. K. Nandi and E. E. Azzouz, "Algorithms for automatic modulation recognition of communication signals," IEEE Transactions on Communications, vol. 46, no. 4, pp. 431-436, 1998.

[9] Z. Fucai, H. Yihua, and H. Shiqi, "Classification using wavelet packet decomposition and support vector machine for digital modulations," Journal of Systems Engineering and Electronics, vol. 19, no. 5, pp. 914-918, 2008.

[10] P. Li, F. Wang, and Z. Wang, "Algorithm for modulation recognition based on high-order cumulants and subspace decomposition," in Proceedings of the 8th International Conference on Signal Processing, ICSP 2006, November 2006.

[11] M. R. Mirarab and M. A. Sobhani, "Robust modulation classification for PSK/QAM/ASK using higher-order cumulants," in Proceedings of the 6th International Conference on Information, Communications and Signal Processing, ICICS, December 2007.

[12] M. Pedzisz and A. Mansour, "Automatic modulation recognition of MPSK signals using constellation rotation and its 4th order cumulant," Digital Signal Processing: A Review Journal, vol. 15, no. 3, pp. 295-304, 2005.

[13] B. Ramkumar, T. Bose, and M. S. Radenkovic, "Robust multiuser automatic modulation classifier for multipath fading 
channels," in Proceedings of the IEEE Symposium on New Frontiers in Dynamic Spectrum, DySPAN 2010, April 2010.

[14] D. C. Chang and P. K. Shih, "Cumulants-based modulation classification technique in multipath fading channels," IET Communications, vol. 9, no. 6, pp. 828-835, 2015.

[15] V. Orlić and M. L. Dukić, "Algorithm for automatic modulation classification in multipath channel based on sixth-order cumulants," in Proceedings of the 9th International Conference on Telecommunication in Modern Satellite, Cable, and Broadcasting Services (TELSIKS '09), pp. 423-426, IEEE, Niš, Serbia, October 2009.

[16] M. S. Mühlhaus, M. Öner, O. A. Dobre, and F. K. Jondral, "A low complexity modulation classification algorithm for MIMO systems," IEEE Communications Letters, vol. 17, no. 10, pp. 18811884, 2013.

[17] M. W. Aslam, Z. Zhu, and A. K. Nandi, "Automatic modulation classification using combination of genetic programming and KNN," IEEE Transactions on Wireless Communications, vol. 11, no. 8, pp. 2742-2750, 2012.

[18] A. K. Nandi and E. E. Azzouz, "Modulation recognition using artificial neural networks," Signal Processing, vol. 56, no. 2, pp. 165-175, 1997.

[19] M. L. D. Wong and A. K. Nandi, "Automatic digital modulation recognition using artificial neural network and genetic algorithm," Signal Processing, vol. 84, no. 2, pp. 351-365, 2004.

[20] E. E. Azzouz and A. K. Nandi, "Automatic identification of digital modulation types," Signal Processing, vol. 47, no. 1, pp. 55-69, 1995. 


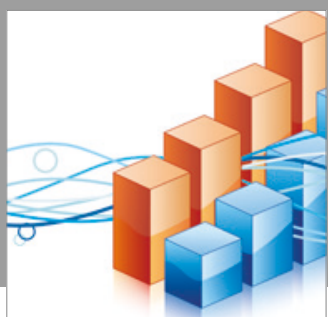

Advances in

Operations Research

vatersals

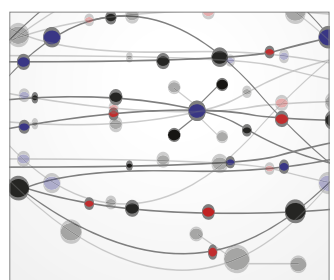

\section{The Scientific} World Journal
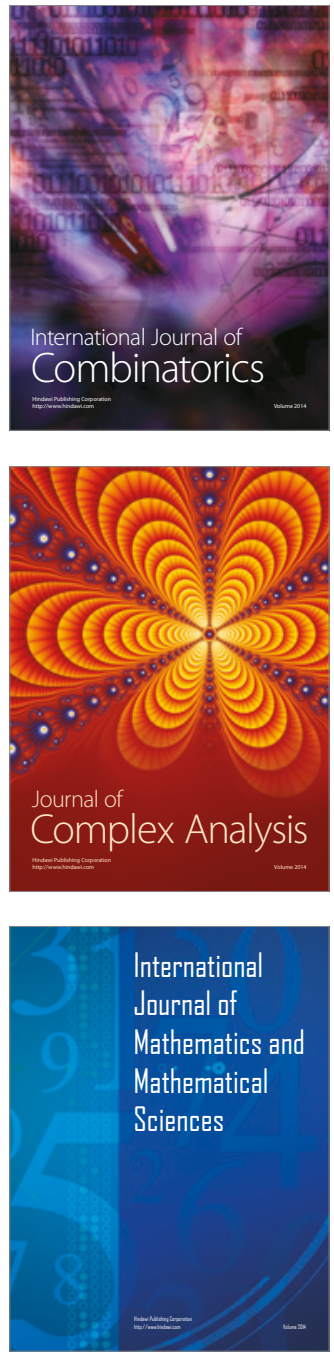
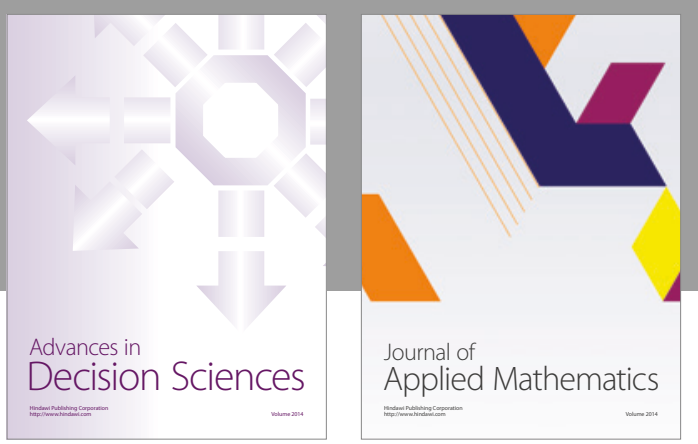

Algebra

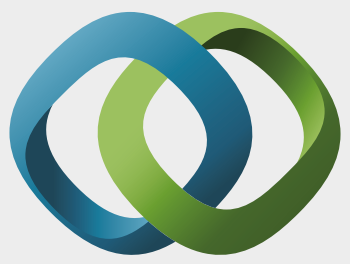

\section{Hindawi}

Submit your manuscripts at

https://www.hindawi.com
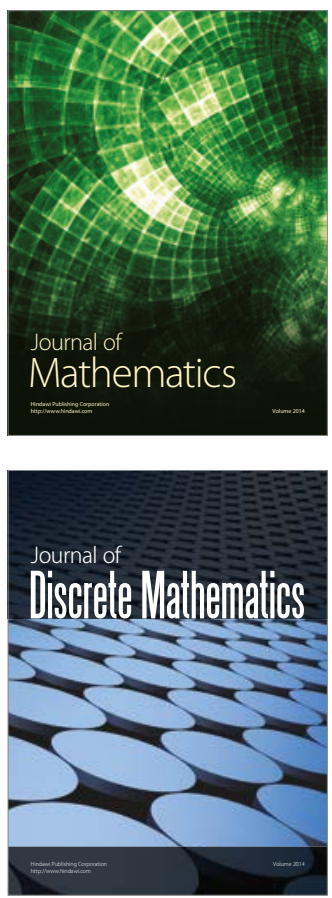

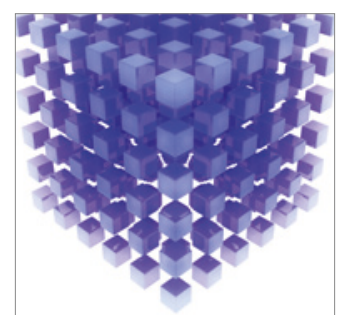

Mathematical Problems in Engineering
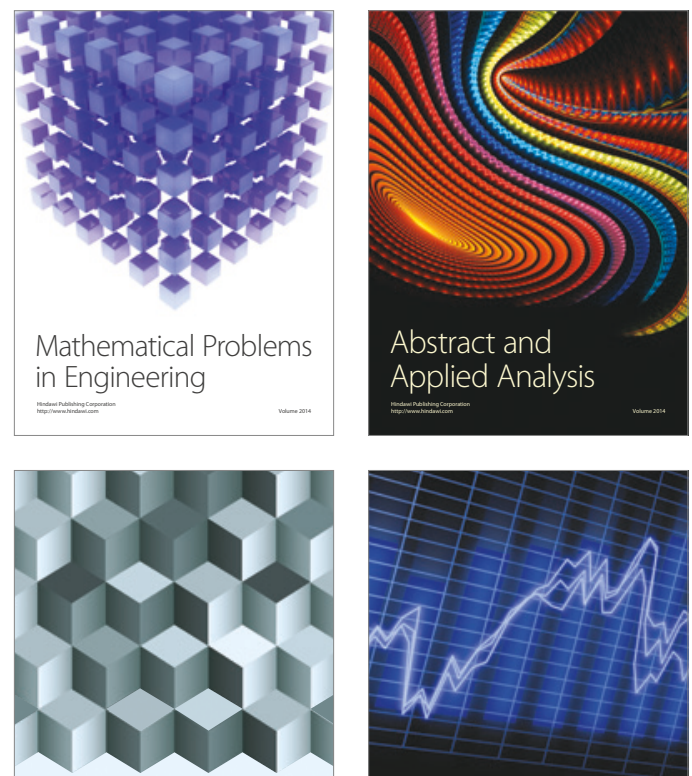

Journal of

Function Spaces

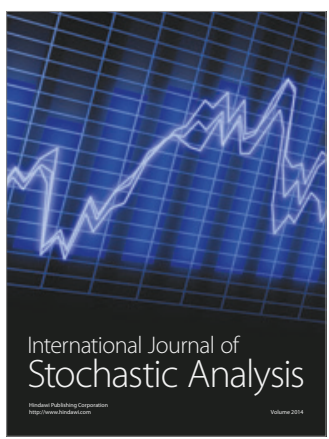

Probability and Statistics
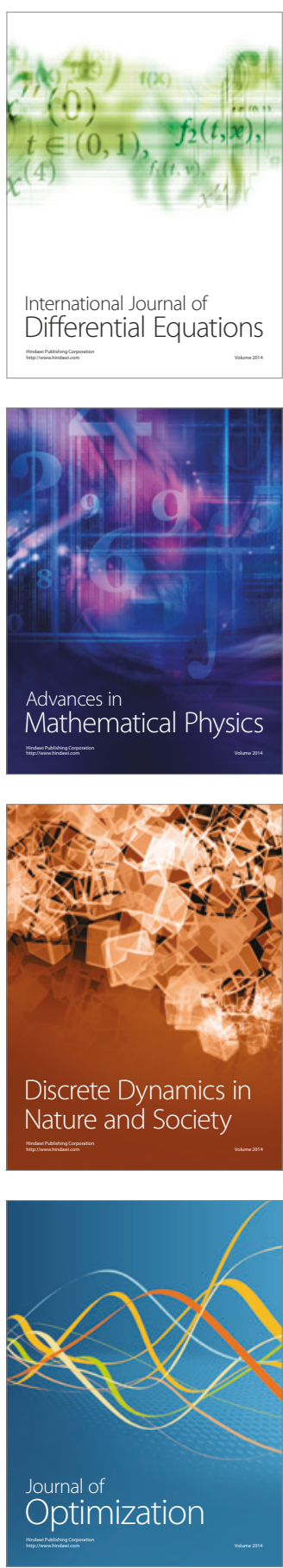